\section{Another notch on cancer's belt}

\section{By Tim Fulmer, Senior Writer}

Italian researchers have identified peptidyl prolyl isomerase as a new player in the Notch signaling pathway that could be targeted to help treat cancer. ${ }^{1}$ The findings potentially open a new avenue for cancer therapies with fewer side effects than compounds that hit other members of the Notch pathway.

Signaling through the Notch family of receptors helps regulate cell fate and cell proliferation in many developmental processes, ${ }^{2}$ and dysfunction of Notch signaling has been implicated in multiple cancers. ${ }^{3-5}$

Pharmas and biotechs have cancer compounds in the clinic targeting $\gamma$-secretase, a pivotal player in the Notch signaling pathway. Blocking $\gamma$-secretase prevents release of a Notch-derived peptide called Notch intracellular domain (NICD), which translocates to the nucleus and potentially activates transcription of oncogenes (see Figure 1, "Pinning down Notch in cancer").

$\gamma$-secretase is also associated with Alzheimer's disease (AD), in which blocking the target limits generation of extracellular $\beta$-amyloid peptides that underlie disease pathology.

For both indications, inhibiting $\gamma$-secretase is associated with off-target toxicities, especially in the GI tract. Thus, some cancer researchers have been looking for other ways to target the Notch signaling pathway.

In a paper in Nature Cell Biology, a group at the University of Trieste reported that one alternative could be targeting molecules that directly or indirectly increase $\gamma$-secretase activity in cancer rather than targeting $\gamma$-secretase itself.

Initial in vitro binding studies showed that NOTCH1 (Notch homo$\log 1$ translocation-associated 9 (Drosophila)) bound PIN1 (protein (peptidylprolyl cis/trans isomerase) NIMA interacting 1), an enzyme that plays a role in multiple signaling processes and has been implicated in cancer and other diseases. ${ }^{6,7}$

Having established a direct interaction between the two proteins, the researchers set out to determine whether PIN1 influenced signaling through NOTCH1.

In human epithelial breast cells, overexpression of PIN1 led to higher levels of NICD than those seen using mock expression vector $(p<0.01)$. In a human breast cancer cell line, small interfering RNA knockdown of PIN1 significantly decreased levels of NICD compared with those seen using control siRNA $(p<0.005)$.

Those findings suggested that by interacting with NOTCH1 at the cell membrane, PIN1 indirectly increased the activity of $\gamma$-secretase, raising levels of the NOTCH1-derived peptide and potentially activating downstream oncogenes.

In a mouse xenograft model of human breast cancer, small hairpin RNA knockdown of PIN1 significantly decreased tumor growth compared with the effects of using control shRNA $(p<0.0006)$.

A second set of experiments revealed that PIN1 increases processing of NOTCH1 at the membrane and that NICD can upregulate PIN1 gene expression in the nucleus. This sets up a positive-feedback loop that increases intracellular levels of NICD and constitutively activates oncogenes in the nucleus.

The researchers also found that PIN1 overexpression correlated with higher levels of NOTCH1 activation in a human breast tumor tissue microarray.

\section{More model behavior}

Two key questions are whether targeting PIN1 will have value across multiple cancer types that have dysfunctional Notch signaling and whether PIN1 is also consistently overexpressed in those cancers in humans.

"While the in vivo data showing xenograft growth inhibition following PIN1 knockdown are intriguing, they represent a single experiment using one breast cancer xenograft cell line. It will be important to validate those findings in additional cancer cell lines and transgenic cancer models," said Charles Eberhart, associate professor of pathology, ophthalmology and oncology and chief of ophthalmic pathology at The Johns Hopkins University School of Medicine.

Similarly, University of Michigan's Max Wicha said that "it would be useful to look at PIN1 levels in animal models of breast and other cancers associated with upregulated Notch signaling to see how closely PIN1 levels correlate with activation of the Notch pathway in multiple tumor types."

Wicha is a professor of oncology at the University of Michigan Medical School, director of the University of Michigan Comprehensive Cancer Center, and cofounder of cancer company OncoMed Pharmaceuticals Inc.

In addition to looking at various tumor types, Thomas Cirrito, director of operations at Stemline Therapeutics Inc., wanted to see data on PIN1-Notch interactions in the three general tumor compartments-bulk tumor cells, cancer stem cells and tumor stroma cells.

"Blocking PIN1 activity might be more therapeutically advantageous in one compartment compared with another," he noted. "In the paper they show an effect on proliferation of bulk tumor cells. However, Notch signaling has been implicated in cancer stem cells, and it might be that PIN1 inhibitors are also capable of blocking self-renewal of those cells. That hypothesis could be tested using xenograft models similar to the one used in the paper."

Stemline has two small molecule $\gamma$-secretase inhibitors in preclinical testing to treat brain cancer. SL-301 and SL-302 both target Notch signaling in cancer stem cells.

Giannino Del Sal, corresponding author on the paper, told SciBX that "by using transgenic animal models with deregulated NOTCH1, 


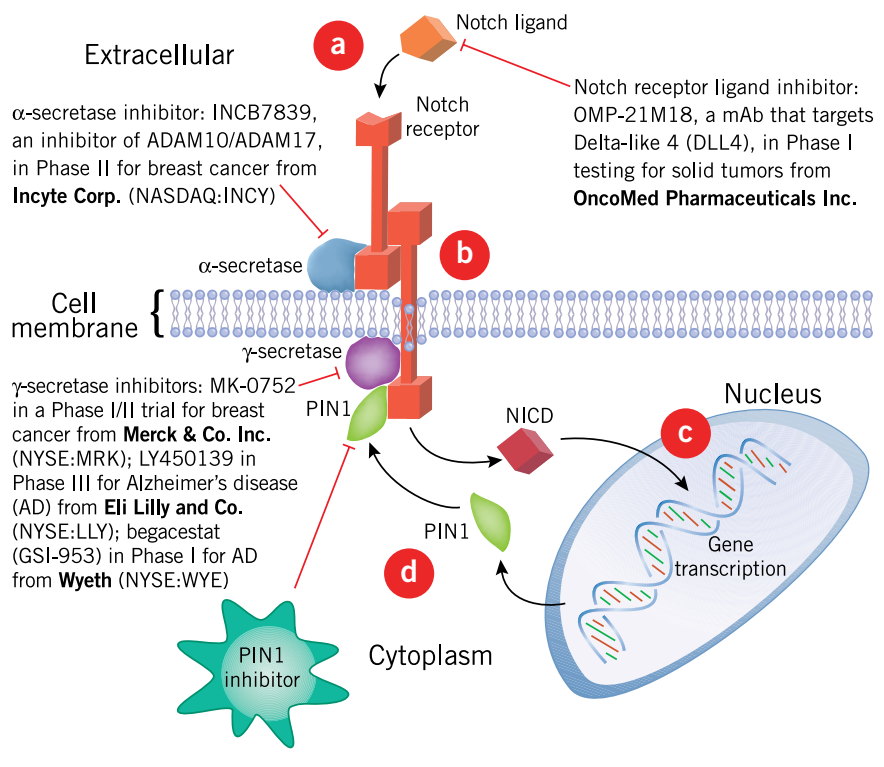

Figure 1. Pinning down Notch in cancer. Notch receptor pathway signaling plays an important role in many developmental processes, and aberrant activation of that pathway has been linked to cancer. A paper published in Nature Chemical Biology suggests that inhibiting PIN1 (peptidyl prolyl isomerase NIMAinteracting 1) could offer a new approach to treating cancer via the Notch pathway. ${ }^{1}$

[a] At the cell surface, Notch receptor ligands such as Jagged and Delta-like proteins bind to the transmembrane Notch receptor.

[b] Interaction between the Notch receptor and its ligand triggers a sequence of proteolytic cleavage events. First, on the extracellular side of the membrane, ADAM metallopeptidase domain 17 (ADAM17; TACE) or ADAM10-both $\alpha$-secretase enzymes - cleave the Notch receptor, generating a substrate for the $\gamma$-secretase complex inside the cell. Second, the $\gamma$-secretase complex cleaves an intracellular portion of the Notch receptor, generating the Notch intracellular domain (NICD).

[c] NICD translocates to the nucleus, where it interacts with transcription factors to upregulate expression of genes. In cancer, NICD can help induce the expression of oncogenes. In the Nature Chemical Biology paper, researchers reported that one of the genes upregulated by NICD in cancer is PIN1.

[d] The PIN1 protein returns to the cytoplasm, where it can interact directly with the Notch receptor to increase production of NICD by $\gamma$-secretase. This sets up a positive-feedback loop that generates ever increasing amounts of NICD and could constitutively activate oncogenes in the nucleus.

Inhibiting PIN1 could block the positive-feedback loop, prevent activation of oncogenes and slow or prevent progression of some cancers. PIN1 inhibitors have not yet entered the clinic for any indication. A number of companies are targeting other components of Notch signaling, including the Notch ligand, $\alpha$-secretase and $\gamma$-secretase. we hope to be able to further study the in vivo effects of inhibiting PIN1 in breast and other cancers."

Using those cancer models, the researchers also hope to see if the NOTCH1-PIN1 interaction holds for other members of the Notch receptor family, said Del Sal, who is professor of molecular oncology at University of Trieste and a researcher at the National Laboratory of the Interuniversity Consortium for Biotechnologies.

Del Sal also wants to look at PIN1 and NOTCH1 levels in human tumors to determine how increased expression of both proteins could potentially influence cancer prognosis.

Lucio Miele of Loyola University Chicago said data on the protein levels will be critical.

"This will give a clearer picture of how well PIN1 expression correlates with Notch activity in clinical specimens," he said. "PIN1 expression alone and in association with the Notch receptor, the Notch receptor ligand and Notch target genes could be correlated with clinical parameters like tumor size, tumor grade, lymph node status and disease-free survival. That should help determine whether PIN1 is an independent prognostic indicator and/or whether it strengthens the prognostic predictive value of other Notch pathway components in cancer."

To get those data, he told SciBX, "larger numbers of patients with different subtypes of breast cancer now need to be studied for PIN1 expression and expression of Notch target genes."

Miele is professor of pathology, pharmacology and experimental therapeutics at Loyola University Medical Center and director of Breast Cancer Preclinical Research at Cardinal Bernardin Cancer Center at Loyola University.

\section{Taking toxicity down a notch}

If PIN1 inhibitors do find a home in the arsenal of cancer therapeutics, the likely starting point would be in combination with $\gamma$-secretase inhibitors and other compounds that target the Notch signaling pathway. Such combinations might allow for lower doses of side effect-laden compounds that directly target Notch signaling.

The authors of the Nature Cell Biology paper reported that treating a human breast cancer cell line with $2 \mu \mathrm{M}$ DAPT, a $\gamma$-secretase inhibitor, plus PIN1 siRNA significantly decreased cell growth compared with what was seen using a $10 \mu \mathrm{M}$ dose of DAPT plus mock siRNA $(p<0.006)$.

"Gut toxicity associated with $\gamma$-secretase inhibitors is one of the most pressing issues with using those compounds to target Notch signaling in cancer," said Eberhart. "The fact that PIN1 inhibition could potentially reduce the doses of $\gamma$-secretase inhibitors is exciting, as it might make amelioration of some of those side effects possible."

Eberhart did caution that PIN1, like $\gamma$-secretase, has multiple targets in addition to Notch, and inhibiting the enzyme could also result in off-pathway effects.

OncoMed is betting that a better way to avoid toxicity is to target the Notch receptor itself and avoid meddling in downstream processing steps involving $\gamma$-secretase and PIN1. The company has generated antibodies that block interactions between Notch receptors and their ligands to prevent aberrant Notch signaling in cancer.

"Because of the way signaling pathways intersect, intracellular targets like PIN1 and $\gamma$-secretase are rarely, if ever, dedicated to a single pathway. Thus, targeting those molecules is not going to be as selective 


\section{TARGETS \& MECHANISMS}

as targeting receptor-ligand interactions on the cell surface," said Tim Hoey, VP of cancer biology at OncoMed.

OncoMed's OMP-21M18, a mAb that targets Notch receptor ligand DLL4, is in Phase I testing to treat solid tumors. According to Hoey, the antibody has shown no GI toxicity in animals and patients.

Another potential strategy for decreasing the gut toxicity of $\gamma$ secretase inhibitors could involve combining them with glucocorticoids. Last month, researchers at Columbia University and colleagues published data showing that a $\gamma$-secretase inhibitor plus glucocorticoid dexamethasone decreased tumor mass and increased survival of murine $\mathrm{T}$ cell acute lymphoblastic leukemia (T-ALL) models compared with what was seen using a $\gamma$-secretase inhibitor alone. ${ }^{8}$

\section{Pinning down inhibitors}

Even if PIN1 is established as a viable target in cancers with dysfunctional Notch signaling, a remaining hurdle will be designing PIN1 inhibitors.

"There are no PIN1 inhibitors out there yet with sufficient affinity and specificity to make them useful for targeting the enzyme in a therapeutic setting," said Kun Ping Lu, professor of medicine at Harvard Medical School. "The two PIN1 inhibitors that have been characterized in vitro - the natural product juglone and the small molecule $\mathrm{PiB}$ - have poor specificity and low potency that significantly reduce their therapeutic value. The ideal therapeutic here would be highlyspecific, small molecule inhibitors with nanomolar affinity for PIN1 that can enter cancer cells but not cross the blood-brain barrier to cause potential neuronal toxicity."

Lu was a cofounder of Pintex Pharmaceuticals Inc., a developer of small molecule PIN1 inhibitors. Vernalis plc acquired the PIN1 IP in 2005, and its PIN1 program remains at "a very early stage," according to spokesperson Annabel Entress.

Notch-targeting combination therapies have reached in the clinic.

Wicha and colleagues at the Baylor College of Medicine and DanaFarber Cancer Institute are running a Phase I trial (NCT00645333) of MK-0752, a $\gamma$-secretase inhibitor from Merck \& Co. Inc., in combination with docetaxel to treat advanced metastatic breast cancer. Miele and colleagues are conducting a pilot trial (NCT00756717) of MK-0752 in combination with letrozole or tamoxifen to treat early-stage estrogen receptor-positive breast cancer.

Del Sal told SciBX the findings in the Nature Cell Biology paper are not patented.

Fulmer, T. SciBX 2(5); doi:10.1038/scibx.2009.168

Published online Feb. 5, 2009

\section{REFERENCES}

1. Rustighi, A. et al. Nat. Cell Biol.; published online Jan. 18, 2009; doi: $10.1038 / \mathrm{ncb} 1822$

Contact: Giannino Del Sal, University of Trieste, Trieste, Italy e-mail: delsal@Incib.it

2. Bray, S. Nat. Rev. Mol. Cell Biol. 7, 678-689 (2006)

3. Radtke, F. \& Raj, K. Nat. Rev. Cancer 3, 756-767 (2003)

4. Shih, I.-M. \& Wang, T.-L. Cancer Res. 67, 1879-1882 (2007)

5. Garber, K. J. Natl. Cancer Inst. 99, 1284-1285 (2007)

6. Lu, K. \& Zhou, X. Nat. Rev. Mol. Cell Biol. 8, 904-916 (2007)

7. Yeh, E. \& Means, A. Nat. Rev. Cancer 7, 381-388 (2007)

8. Real, P. et al. Nat. Med. 15, 50-58 (2009)

\section{COMPANIES AND INSTITUTIONS MENTIONED}

Baylor College of Medicine, Houston, Texas

Cardinal Bernardin Cancer Center at Loyola University,

Chicago, III.

Columbia University, New York, N.Y.

Dana-Farber Cancer Institute, Boston, Mass.

Harvard Medical School, Boston, Mass.

The Johns Hopkins University School of Medicine, Baltimore, Md. Loyola University Chicago, Chicago, III.

Loyola University Medical Center, Chicago, III.

Merck \& Co. Inc. (NYSE:MRK), Whitehouse Station, N.J.

National Laboratory of the Interuniversity Consortium for Biotechnologies, Trieste, Italy

OncoMed Pharmaceuticals Inc., Redwood City, Calif.

Pintex Pharmaceuticals Inc., Watertown, Mass.

Stemline Therapeutics Inc., New York, N.Y.

University of Michigan, Ann Arbor, Mich.

University of Michigan Comprehensive Cancer Center,

Ann Arbor, Mich.

University of Michigan Medical School, Ann Arbor, Mich.

University of Trieste, Trieste, Italy

Vernalis plc (LSE:VER), Reading, U.K. 